fit athletes training at both lower elevations and elevations greater than 5000 feet. Military population rates of injury have been consistently lower than civilian sectors in previous studies. Nevertheless, no large-scale studies of this type have looked at altitude and the effects of hydration on SCT-related complications. The military uses an aggressive hydration and work/rest protocol for all soldiers.

Objective.-The investigators hypothesized that hydration and work/rest protocols may have led to lower rates of injury in soldiers with SCT exposed to altitude.

Methods.-We conducted a retrospective review of a 2800 soldier cohort using blinded data of injuries occurring in participants with pre-identified SCT during a series of 28-day Marine Mountain Warfare training events conducted in Bridgeport, CA from May 2010-Feb 2012. All soldiers with SCT enrolled in the course were included in the study and their injury patterns followed. We compared their patterns with those of the non-SCT population.

Results.-Out of the 2800 soldiers, 25 had SCT, but only 1 of the SCT group had an altitude-related complication (acute mountain sickness). Surprisingly, despite a reported clear predilection for complications secondary to SCT, altitudespecific illnesses were rare and only occurred once out of 25 participants (4\%). The rate of injuries was much lower than previous civilian studies. There were no deaths in either the study group or the control group.

Conclusions.-Medical providers must be prepared to recognize possible SCT-related complications at elevations above 510,000 feet, but also recognize that it may be far more likely that their soldiers with SCT will more likely have a wide range of routine illnesses and injuries that are not altitude related.

Ian C. May, MD Garrett Ripoll, DO Ian Wedmore, MD

Carl Skinner, MD

Darren Thomas, MD

Owen McGrane, MD

San Diego, CA, USA

\section{6-Minute Walk Test as a Predictor of Summit Success on Denali}

Introduction.-A growing number of climbers attempts mountains over 5,500 meters each year. The rescue of climbers from these elevations is dangerous and expensive, with each rescue on Denali averaging over $\$ 20,000$. The 6-minute walk test (6MWT), previously tested on Aconcagua, is an inexpensive and easily reproducible screening test for summit success that may provide early detection for those requiring rescue.

Objective.-The purpose of this study was to validate the $6 \mathrm{MWT}$ at $14,000 \mathrm{ft}(4,267 \mathrm{~m})$ on Denali, Alaska.

Methods.-This was a prospective observational cohort study of healthy adults between the ages of 18 and 65 who had been at 14,000 ft for less than 24 hours in June, 2013. Vital signs (VS) were measured before and after the 6MWT.
Subjects attempted to summit at their own pace and, at the time of descent, returned to the study tent and completed a Lake Louise AMS Questionnaire (LLQ) and reported maximum elevation reached.

Results.-One hundred and twenty-one participants enrolled in the study. Complete data was collected on 100 subjects (86\% response rate) of whom 62\% summited. Univariate analysis revealed that there was no association between any resting or post-exercise VS and summit success. Specifically, there was no significant difference between the post-exercise oxygen saturation in summiters $(\mathrm{S}, 74.6 \%)$ and non-summiters (NS, 74.05\%), which differed by $-0.54 \%$ (95\% CI: $-0.93 \%$ to $-0.13 \%, P=.53$ ). The 6-minute walk test distance (6MWTD) revealed marginal significance with $2025 \mathrm{ft}(\mathrm{S})$ and $1855 \mathrm{ft}$ (NS) for a difference of -170 feet (95\% CI: $-247.6 \mathrm{ft}$ to $-91.39 \mathrm{ft}, P=.048$; OR: $1.000 ; 95 \%$ CI: 1.000 to 1.002$)$.

Conclusions.- This study showed that the post-exercise oxygen saturation does not correlate with summit success. The distance a subject covered in 6 minutes, however, may be a better measure of functional status at altitude.

\author{
Katherine M. Shea, MD \\ Eric R. Ladd, MD \\ Grant S. Lipman, MD \\ Patrick Bagley \\ Elizabeth Pirrotta \\ Hurnan Vongsachang \\ $\mathrm{N}$ Ewen Wang, MD \\ Paul S. Auerbach, MD \\ Stanford, CA, USA
}

\section{Effectiveness of Preventive Search and Rescue: Illness and Injury Prevention and Fiscal Impact}

Introduction.-Grand Canyon National Park Rangers respond frequently to calls for assistance in the backcountry. The Preventive Search and Rescue Team (PSAR) seeks to educate the hiking public in Grand Canyon and reduce the number of hikers requiring assistance.

Objectives. - This study investigates the effectiveness of the PSAR Team by examining its impact on heat-related search and rescues (SARs) and non-heat-related SARs, in addition to its fiscal impact.

Methods.-Historical data collected on heat-related and non-heat-related SARs was used to develop predictive models about pre-PSAR SAR rates. These models were then compared to recent data collected by the PSAR Team on heat-related and non-heat-related SARs that occurred after the inception of the PSAR Team.

Results.-A $42 \%$ reduction in heat-related SARs, $21 \%$ reduction in non-heat-related SARs, and an annual fiscal savings of at least $\$ 300,000$ were observed after the implementation of the PSAR Team.

Conclusions.- The PSAR Team has a great impact on the occurrence of SARs, both heat and non-heat related. By 
effectively reducing the number of responses required by rangers in Grand Canyon National Park, the PSAR Team saves the National Park Service over \$300,000 annually.

Christian Malcolm, MA/NREMT-P Hannah Heinrich, PhD Emily Pearce, BS/NREMT-P Grand Canyon National Park, AZ, USA

\section{Physiological Temperature Thresholds and Heat-Related Hiker Assists: Preventive Search and Rescue in Grand Canyon National Park}

Introduction.--Heat-related hiking emergencies in Grand Canyon National Park occur frequently and place great demand on resources. The Preventive Search and Rescue Team (PSAR) perform approximately 750 hiker assists and 300 search and rescue (SAR) missions annually.

Objectives.-This study examines hiker assist frequencies in correlation with maximum daytime temperatures in Grand Canyon National Park, with the goal of gathering information to improve the PSAR Team's strategy: to better utilize limited resources and provide a safer and more enjoyable National Park experience.

Methods.-Data was collected for this prospective study by PSAR rangers in 2011 and 2012 for all hikers who required assistance while hiking in Grand Canyon National Park. Inclusion criteria were created for heat-related hiker assists. The number of preventive actions, heat-related and non-heatrelated hiker assists was compared to average maximum daytime temperatures in Grand Canyon.

Results.-Heat-related hiker assists increased by $61.5 \%$ when maximum daytime temperatures were $\geq 95^{\circ} \mathrm{F}$. No correlation was found between non-heat-related hiker assists and temperature. Preventive actions remained consistent across all temperature ranges.

Conclusions.-The incidence of heat-related hiker assists is significantly higher at or above a temperature threshold of $95^{\circ} \mathrm{F}$, identifying a critical inflection point for temperaturerelated hiker safety. High temperatures increase risk for heatrelated illness or potential death. Individuals hiking in Grand Canyon National Park may therefore benefit from increased caution while hiking in temperatures at or exceeding $95^{\circ} \mathrm{F}$. Additionally, Preventive Search and Rescue rangers may benefit from heightened awareness, preparedness, and staffing on days exceeding this temperature threshold achieving higher safety standards for personnel.

Christian Malcolm, MA/NREMT-P

Hannah Heinrich, PhD

Emily Pearce, BS/NREMT-P

Grand Canyon National Park, AZ, USA

\section{Chaos in the Wilderness: Lifesaving Perfor- mance of Medical Teams in Austere Environments}

Introduction.-The provision of life-saving medical care in austere environments requires individuals working in teams to rescue patients, yet few studies measure trainee performance in these environments.

Objectives.-Our goal was to develop a competency-based curriculum for patient care providers using a chaotic, multipatient, and environmentally challenging environment.

Methods.-A 5-hour wilderness/emergency preparedness training (EPT) curriculum was developed by a statewide panel of experts including 9 learning objectives, 18 competencies and 34 performance objectives. Following brief didactic and small group sessions, inter-professional teams of 5-6 trainees were observed in a large patient simulator designed to recreate environmentally challenging (ie, flood evacuation), multipatient scenarios using trained patient actors. Successful completion of 16 individual and 18 team performance objectives were assessed by trained observers. Prior to training, team members completed a 24-question knowledge assessment, a demographic survey and a comfort level self-assessment. Following training, trainees repeated the 24 questions, selfassessment, and a course assessment.

Results.-One-hundred ninety-five participants completed the course between November 2012 and August 2013, including $90(46.1 \%)$ health professional students, 60 (30.8\%) physicians, $32(16.4 \%)$ nurses, $5(2.6 \%)$ health administrators, $3(1.5 \%) \mathrm{EMS} /$ fire personnel, $3(1.5 \%)$ environmental engineers, and $2(1.0 \%)$ law enforcement officials. Thirty-eight $(19.5 \%)$ reported $>3$ hours of wilderness/EPT each year while $157(80.5 \%)$ reported $\leq 3$ hours of yearly EPT. One-hundred ninety-five (73.3\%), 139 (52.2\%) and 66 (33.8\%) participants completed the pretest, posttest, and course assessment. The average percentage of correct answers between the pretest and posttest increased from 47.4 to 77.8 . $66(100 \%)$ reported the course relevant to care providers and 61 (92.4\%) highly recommended the course.

Conclusions.-Our competency-based wilderness/EPT curriculum used a large simulator to measure performance in a chaotic environment. Novice and experienced trainees improved knowledge and highly recommended the course. Further research is needed to assess the impact of the curriculum on individuals and teams.

Lancer A. Scott, MD Julie Teuber, BS Jamal Jones, BS Judy Staub, BS Andrew Seymore, BS Charleston, SC, USA 